\title{
Experiment Teaching Reform of Principle of Computer Composition
}

\author{
Tao Chi \\ Shanghai Ocean University \\ College of Information Technology \\ Shanghai, China \\ tchi@shou.edu.cn \\ Xiaojing Shen \\ Shanghai Ocean University \\ College of Information Technology \\ Shanghai, China \\ xjshen@shou.edu.cn
}

\author{
Guangpu Cao \\ Shanghai Ocean University \\ College of Information Technology \\ Shanghai, China \\ guangfucao@hotmail.com \\ Haowei Yan \\ Shanghai Ocean University \\ College of Information Technology \\ Shanghai, China
}

\begin{abstract}
Experiment teaching content of Principle of Computer Composition is complicated and abstract, mixing with a lot of specious concept. It is difficult for students to master the operating mechanism of a real computer system. This paper expounds how to combine the project to improve their experiment teaching, and ensure the students really develop an actual computer system. Such experiments can greatly improve the students' practical ability and obtain more profound understanding of the CPU structure.
\end{abstract}

Keywords-principles of computer composition; experiment reform; experiment teaching; project teaching method

\section{INTRODUCTION}

Principle of computer composition is a core course, which forms a connecting link between the preceding and following courses in computer major. Because the course content is complex, and mix with a lot of specious concept, it can only be combined with the actual project to understand how a computer system works, and grasp the key and difficult points of this course ${ }^{[1]}$.

"Experiment teaching of Principle of computer composition" is an experiment course for computer science and technology, in the beginning, the experiment box of computer constitution principle was used to carry out the experiment, through the testing and operation over the past few years, there are many disadvantages in the experiment box:

- The experimental method is out of line with the prior hardware course. There has been a close link between the hardware courses, due to different courses use different experiment boxes, it is difficult to establish contact among courses, and exists the serious phenomenon of theory's separation from

Key courses construction of universities in Shanghai 'Principle of computer composition', and Construction of full English demonstration course for overseas students in Shanghai ' Principles and design of embedded system practice.

- The hardware structure of the experiment box is fixed, the main work of the students is only to plug in the wiring between the corresponding hardware modules, so the experiments are mostly confirmatory experiments, students can't design modules themselves, all experiments are difficult to break through the limitations of the experiment box.

- The number of experiment boxes is difficult to satisfy the students' increasing needs, and laboratory hardware investment is relatively large, so the number of experiment boxes was inadequate without sufficient funds.

- Because of the particularity of the experiment boxes, the whole experiment box is scrapped in the event of a system failure; it's difficult to maintain laboratory equipment and apparatus.

To this end, we introduced EDA technology in the "Experiment teaching of Principle of computer composition", The experiment can complete the function design, logic design, performance analysis and function test by computer simulation. Finally, a tutorial file is generated to guide further experiments. Through the application practice in recent years, we used computer simulation software QUARTUS to design the CPU machine; Then, computer simulation software MODELSIM is used to show simulation results. Through such experiments, the students' practical ability is greatly improved, and more profound understand of the CPU structure ${ }^{[2]}$.

\section{CURRICULUM PlanNING}

In order to really understand the composition of a computer system and its internal operating mechanism, students are required to complete the design and debugging 
of a computer system (model machine) in a experimental course. However, it is difficult to achieve this goal, mainly due to the lack of the basic level and practical experience of students, they can't understand CPU architecture. In order to develop an effective "design method" as a guide to achieve the teaching purpose, several key points are considered:

- On the basis of the basic concepts of textbooks, we begin to learn the CPU Design from the underlying hardware.

- Designing the underlying module through computer simulation software QUARTUS, such as register module, operation module design, sequential circuits, controller design, in this way, students not only mastered the basic design of hardware module, but also have an intimate knowledge of the hardware description language.

- Students are required to design the instruction set, including instruction format design specification, the description of each instruction and functional components involved, understanding of CISC / RISC instruction system (the relationship between assembly and machine code).

- Design of algorithm state machine. Design some projects; transform questions in natural language into the sequence flow chart which can be processed by computers.

- As long as the sequence flow chart once formed, it's easy to obtain state function and the output function, accordingly, the corresponding hardware circuit is obtained.

- System simulation design. Through the computer simulation software MODELSIM to design the system simulation, through software debugging to ensure that the whole system can be logically realized, It also provides the basis for following hardware debugging.

\section{PRoject FORMULATION}

\section{A. Basic principles of project formulation}

The most basic and the most critical problem of Project teaching method is project formulation, quality of "project" has a direct impact on the teaching effect, so it's necessary to establish some basic principles of project formulation. Through practice, we believe that the following principles can guide the formulation of the project:

- The project formulation should be based on the teaching objectives, contents and features of the course "principles of computer composition". The experiment content should take the teaching content as the core; It is to make the students solve the problem combining with the teaching content.
- Degree of the project difficulty. The limited time for the course severely affect the project difficulty, and too simple items will affect students' learning initiative, so we should consider the students' ability and class restrictions when we design a project.

- Because of restrictions of experimental conditions, it's necessary to sure students can design and implement the project.

\section{B. Project and contents}

According to practical experience, we consider that the project should include the name of the project, content and requirements. And the number of items should be as large as possible, so that students can choose according to their personal interests. As for the project, we give some examples $^{[3]}$ :

- Fixed-point arithmetic unit: Designing a 16 bit fixed-point arithmetic unit, which can add, subtract, multiply and divide operations of 16 bit binary numbers, and can work in two states of offline/online.

- Sequence generator: designing a sequence generator which can produces 6 beat pulses T0-T6.

- Counter: Designing a 16bit counter, which can realize loop counting function.

- Microprogram controller: Based on the existing teaching machine instruction system, adding 8-10 new instructions, including defining instruction formats and functions. Then debugging the new instruction correctly and add it to the teaching computer hardware system

Take Microprogram controller for example:

TABLE I. SINGLE BYTE INSTRUCTION

\begin{tabular}{|l|l|l|}
\hline Opcode 4bits & Destination register 2bits & Source register 2bits \\
\hline
\end{tabular}

TABLE II. DOUBLE BYTE INSTRUCTION

\begin{tabular}{|l|l|l|}
\hline Opcode 4bits & Destination register 2bits & Source register 2bits \\
\hline & Immediate digital segment 8bits \\
\hline
\end{tabular}

1) Instruction set

The CPU model has 13 instructions, the 0-9 instruction is a single byte instruction, and the $10-13$ instruction is a double byte command.

TABLE III. INSTRUCTION SET

\begin{tabular}{|c|c|c|c|}
\hline Serial & Instruction & Function & Opcode \\
\hline 0 & IN Rd & $\mathrm{IN} \leftarrow \mathrm{Rd}$ & 0000 \\
\hline 1 & OUT Rs & 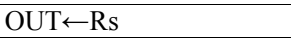 & 0001 \\
\hline 2 & MOV Rd, Rs & $\mathrm{Rd} \leftarrow \mathrm{Rs}$ & 0010 \\
\hline 3 & ADD Rd, Rs & $\mathrm{Rd} \leftarrow \mathrm{Rs}+\mathrm{Rd}$ & 0011 \\
\hline 4 & AND Rd, Rs & $\mathrm{Rd} \leftarrow \mathrm{Rs} \& \mathrm{Rd}$ & 0100 \\
\hline 5 & MUL & $\{\mathrm{HIGH}, \mathrm{LOW}\} \leftarrow \mathrm{RD} * \mathrm{RS}$ & 0101 \\
\hline 6 & STI & Enable interrupt & 0110 \\
\hline
\end{tabular}




\begin{tabular}{|c|c|c|c|}
\hline \multicolumn{4}{|c|}{ Cont. to TABLE III } \\
\hline 7 & CLI & Disable interrupt & 0111 \\
\hline 8 & IRET & Interrupt return & 1000 \\
\hline 9 & HLT & Machine halt & 1001 \\
\hline 10 & LDI & $\mathrm{Rd} \leftarrow$ Immediate number & 1010 \\
\hline 11 & LAD & $\mathrm{Rd} \leftarrow \mathrm{MEM}$ & 1011 \\
\hline 12 & STA & MEM $\leftarrow$ Rs & 1100 \\
\hline 13 & JMP target & PC $\leftarrow$ Address & 1101 \\
\hline 14 & JMP target & $\begin{array}{l}\text { Conditional transfer: } \\
\text { If FC==1'b1, } \\
\text { PC } \leftarrow \text { Immediate number, } \\
\text { Otherwise, the PC is not } \\
\text { modified. }\end{array}$ & 1110 \\
\hline
\end{tabular}

2) $C P U$ structure

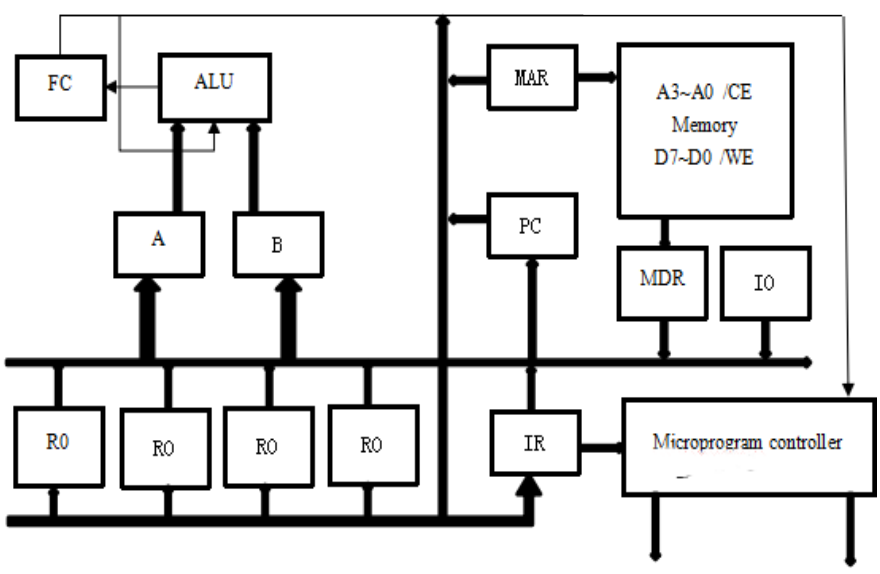

Fig. 1. CPU Structure

3) ModelSim can be used to verify the CPU working situation

Students can also put forward other projects according to the knowledge they have. Obviously, it is good for developing students' independent thinking and creative ability.

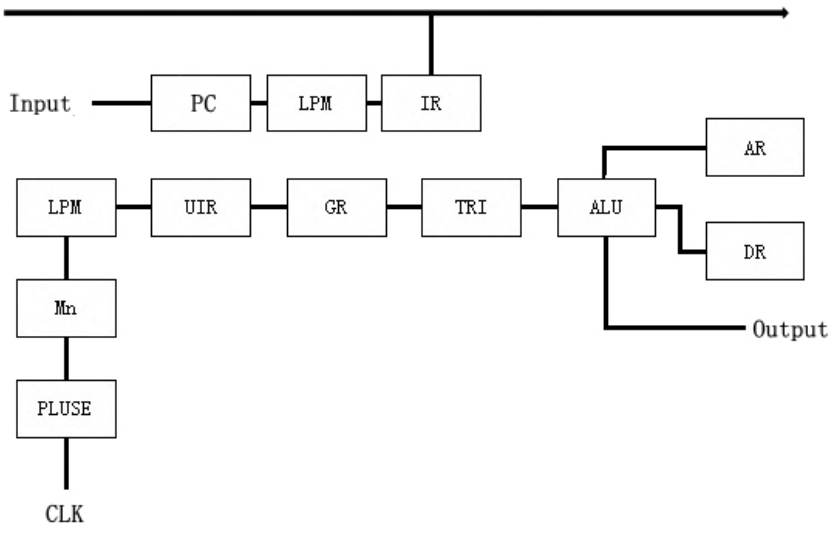

Fig. 2. Device Diagram in Quartus

\section{CONCLUSION}

Through the practice of "project teaching method", the following beneficial experiences are obtained:

- From the formulation and implementation of the project to the evaluation of the project completion, students are involved in it and fully reflect the students' Learning initiative. And in the process, it also improves the students' enthusiasm and efficiency in learning the principles of computer composition.

- After adopting this teaching method, students arrange the experiment time and content independently according to their individual time, and students' design and experimental ability has been effectively improved.

- Through the practice of the course, the method, content and practical experiences of "project teaching method" are accumulated, which lays a foundation for other courses.

\section{ACKNOWLEDGMENT}

In this paper, the research was sponsored by 2017 annual key curriculum construction project of Municipal Education Commission (Principles of Computer Composition) and Construction of demonstrative courses for foreign students in Shanghai in 2015 (Embedded System Design and Application).

Thanks also to Shanghai Ocean University for allowing us to build a computer science and technology hardware course group teaching team.

\section{REFERENCES}

[1] Hou H X. The Study on Improving the Quality of Teaching in "Experiment teaching of Principle of computer composition"[J]. Computer Education, 2009.

[2] Zheng G Z, Liu Q M. The Study of Experiment Teaching Reform Based on computer composition Principle[J]. Computer Knowledge \& Technology, 2011.

[3] Ling-Hua L I. Discussion and Practice on the Experiment Teaching of the Course of Principle of computer composition[J]. Computer Knowledge \& Technology, 2016.

[4] Luo Yigui. Experimental teaching practice of embedded system based on problem solving. Research and exploration of [J]. Laboratory, 2012, v.31, No.200 (10): 389-391.

[5] Liu Jing. Design and development of embedded teaching experiment platform based on ARM. [D]. University of Electronic Science and technology of China, 2014.

[6] Chen Xi. Research and practice of embedded remote experiment system [D]. Beijing University of Posts and Telecommunications, 2014.

[7] Huang Xiaoling, Duan Wei, Zhao Jianke. Exploration and practice of experimental teaching system of embedded system [J]. Experimental technology and management, 2006, 23 (4): 85-87. 